and saline placebo, in patients with stable hip OA. Small injection volumes of $2 \mathrm{ml}$ were used to avoid joint-lavage effects.

Although in all 101 treated patients (placebo, $n=36$; hyaluronic acid, $n=33$; corticosteroids, $n=32$ ) the injections were well tolerated and there were no serious adverse effects, only those who received corticosteroids showed a moderate (but transient) improvement in pain scores. Corticosteroid injection might, therefore, have a role in acute pain relief for patients with hip OA. None of the groups showed an improvement in any of the outcome measures by the 3-month follow-up. This duration should have been sufficient to show an effect, according to the literature on intra-articular injection therapies, and, therefore, these treatments cannot be recommended as standard therapy for hip OA.

These results rule out a moderate-to-large clinical effect of hyaluronic-acid injection in hip $\mathrm{OA}$, say the authors, although future studies with 100 patients in each treatment group could reveal a small effect size. They speculate that high-molecular-weight hyaluronic acid might have a greater effect than was seen in this study.

\section{Caroline Barranco}

Original article Qvistgard E et al. (2006) Intra-articular treatment of hip osteoarthritis: a randomized trial of hyaluronic acid, corticosteroid, and isotonic saline. Osteoarthritis Cartilage 14: 163-170

\section{Potential predictors of failure to respond to antirheumatic drugs}

A large number of patients fail to respond to disease-modifying antirheumatic drugs (DMARDs), which are a major component of the treatment of rheumatoid arthritis. Failure to respond to these agents is an important clinical issue and is almost impossible to predict at the present time. A team of researchers from the Mayo Clinic conducted a long-term, retrospective, observational study of patients with rheumatoid arthritis, in an attempt to identify which factors predict discontinuation of DMARDs.

The study included 345 patients $(72.8 \%$ female) first diagnosed with rheumatoid arthritis between 1955 and 1995, who were followed for a mean of 15.4 years from diagnosis. Of all the disease characteristics assessed, only high disease activity was associated with an increased risk of discontinuation. Contrary to common assumption, disease duration and the number of previous DMARD treatments were not. Interestingly, the type of DMARD mattered: durations of treatment with either hydroxychloroquine or methotrexate were substantially longer than with any other kind of DMARD. Methotrexate-treatment duration was extended with the addition of folate supplementation. In addition, methotrexate failure was associated with almost twice the likelihood of discontinuing another DMARD.

The authors suggest that it might be possible to identify patients who will later discontinue DMARD treatment, using these predictors. Patients who do not respond to methotrexate are a particularly high-risk group, and could be considered for new therapies. They also recommend that folate supplementation be included in all future methotrexate regimens.

Katherine Sole

Original article Maradit-Kremers $\mathrm{H}$ et al. (2006)

Patient, disease, and therapy-related factors that influence discontinuation of disease-modifying antirheumatic drugs: a population-based incidence cohort of patients with rheumatoid arthritis. J Rheumatol 33: 248-255

\section{Pulmonary arterial hypertension predicts death in diffuse cutaneous systemic sclerosis}

Pulmonary complications, including pulmonary arterial hypertension (PAH) and interstitial lung disease (ILD), are the leading cause of death in patients with diffuse cutaneous systemic sclerosis (dcSSc). About half of dcSSc patients with PAH have pulmonary fibrosis and, therefore, ILD is thought to be a forerunner of PAH. In a retrospective study, Trad et al. found that PAH predicts death in patients with dcSSc independently of ILD: they suggest, therefore, that ILD and PAH have distinct pathophysiologies.

Of the 86 patients with dcSSc included in the study, 52 had ILD, 15 had both ILD and PAH, 3 had isolated PAH and the remainder had dcSSc only. On univariate analysis, survival was substantially worse in patients with $\mathrm{PAH}$, and on multivariate analysis, age and $\mathrm{PAH}$ were the only independent predictors of death.

During the study, 20 patients with ILD (including 8 who also had PAH) were treated with cyclophosphamide. In patients with PAH, 